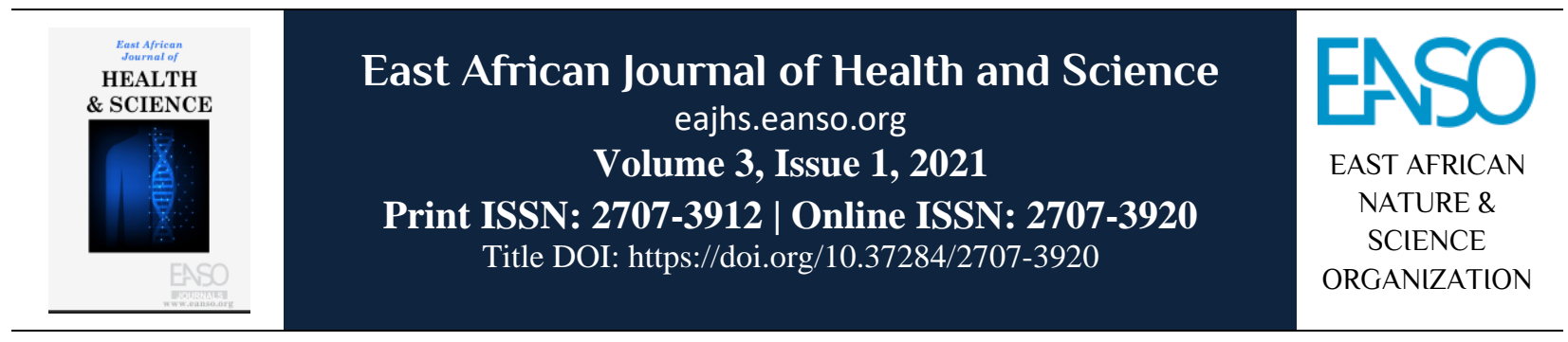

Original Article

\title{
Exploring the Use of Cognitive Approach in Mitigating Unsafe Excreta Disposal in Turkana County, Kenya.
}

\author{
Andrew Rutto Kiptum ${ }^{1 *} \&$ Moturi Adelaide Moraa ${ }^{2}$ \\ ${ }^{1}$ University of Eldoret, P. O. Box 1125-30100, Eldoret, Kenya; \\ ${ }^{2}$ Action for Cheetahs in Kenya, P. O. Box 1611-00606, Nairobi, Kenya; \\ *Author for correspondence email: kiptumandrew@gmail.com.
}

Article DOI: https://doi.org/10.37284/eajhs.3.1.294

\section{Date Published: ABSTRACT}

11 March 2021 We evaluated the influence of induced behavioural change on the community to adopt safe excreta disposal practices. This study was informed by unmerited

Keywords: programs which are majorly provided by most of the health organisations providing

Waterborne

Epidemics,

Triggering,

Health Experts,

Personal Hygiene,

Latrine Use. sanitary facilities rather than effective use which have failed to reduce the persistent occurrence of waterborne epidemics due to unsafe excreta disposal. Information sought in this survey was from two distinct populations of triggered and nontriggered villages of Turkana County, Kenya. Purposive sampling was used in identifying triggered and non-triggered villages, while systematic random sampling was used to administer structured questionnaires to respondents from identified sample units. From analysed data, the results showed latrine users from triggered villages were many compared with non-triggered villages. Again, the variable of education level, which showed to be critical, suggesting to influence latrine use among rural communities irrespective of the triggers. The parameter estimator of public health extension services and information sharing means from the neighbourhood showed to be statistically significant in influencing adoption and diffusion of health knowledge. In conclusion, involving health experts in knowledge diffusion and scaling up formal education among communities influences the adoption of safe excreta disposal practices. The significance of random factor in the OLS model, explains omitted and/or unobserved variables and existences of unclearly explained variables, informs the need for further investigation.

\section{APA CITATION}

Kiptum, A. R., \& Moraa M. A. (2021). Exploring the Use of Cognitive Approach in Mitigating Unsafe Excreta Disposal in Turkana County, Kenya. East African Journal of Health and Science, 3(1), 20-30. https://doi.org/10.37284/eajhs.3.1. 294. 


\section{CHICAGO CITATION}

Kiptum, Andrew Rutto., \& Moturi Adelaide Moraa. 2021. "Exploring the Use of Cognitive Approach in Mitigating Unsafe Excreta Disposal in Turkana County, Kenya.". East African Journal of Health and Science 3 (1), 20-30. https://doi.org/10.37284/eajhs.3.1.294.

\section{HARVARD CITATION}

Kiptum, A. R., \& Moraa M. A. (2021) "Exploring the Use of Cognitive Approach in Mitigating Unsafe Excreta Disposal in Turkana County, Kenya.”, East African Journal of Health and Science, 3(1), pp. 20-30. doi: 10.37284/eajhs.3.1. 294.

\section{IEEE CITATION}

A. R. Kiptum and M. A. Moraa, "Exploring the Use of Cognitive Approach in Mitigating Unsafe Excreta Disposal in Turkana County, Kenya.”, EAJHS, vol. 3, no. 1, pp. 20-30, Mar. 2021.

\section{MLA CITATION}

Kiptum, Andrew Rutto, \& Moturi Adelaide Moraa. "Exploring the Use of Cognitive Approach in Mitigating Unsafe Excreta Disposal in Turkana County, Kenya.". East African Journal of Health and Science, Vol. 3, no. 1, Mar 2021, pp. 20-30, doi:10.37284/eajhs.3.1.294.

\section{INTRODUCTION}

The dichotomous relationship of deaths from waterborne epidemics that results from environmental factors such as inadequacy of basic environmental sanitation is a serious health risk and an affront to human dignity (Izua et al., 2015). The unhygienic environment exposes humans to diseases. Sustainable development goals set in 2015 aims at reducing the number of people without access to clean and basic sanitation. However, statistical evidence shows a worrying trend indicating a high mortality rate, especially to young children who are under five years (Venkataramanan et al., 2017). High costs from diseases that are associated with an unhygienic environment in developing economies show no sign of reduction, despite the numerous sanitation intervention programs (WHO \& UNICEF, 2014). Water-related diseases such as typhoid, diarrhoea, and dysentery, among others, account for about $17 \%$ of the disease burden in developing countries (Izua et al., 2015; Venkataramanan et al., 2017). About $88 \%$ of cases of diarrhoea worldwide often occur during the rainy season and in places with widespread unsafe excreta disposal. While the projection of the population globally that faces the health risks from consumption of polluted water often from open defecation is over 2.6 billion which is nearly $40 \%$, with the majority being found in developing economies (WHO \& UNICEF, 2014).

According to the World Bank - Joint Monitoring Programme Data of 2012, countries in Sub-Sahara to experience high open defecation. In Kenya, open defecation is predominantly in informal urban settlements and rural areas as well as rampantly in arid and semi-arid rural areas (WSP et al., 2013). Arid and semi-arid areas are characterised by scarcity of consumable water and a population that is highly dependent on livestock keeping and to some extensively do practice nomadic lifestyle. Characteristics of households in the study area are often found to settle near places of a watercourse, which allows them to access water from shallow depression or dug shallow wells for domestic and household use. Open defecation is a widespread phenomenon in dry areas of Kenya remains a major health setback, especially during flash floods or rainy seasons. Polluted water from flash floods in a place with scarce consumable water tends to worsen already a most sought commodity. This expose both the human and animal population to diseases and/or aggravating their stakes by putting lives into a perilous state. For instance, the existence of open defecation in a place attracts wild animals that scavenge on human waste which possibly could transmit zoonotic disease (Tessema, 2017).

Despite high publicity of health concerns via promotions aimed at bringing awareness in order to reduce the open defecation menace, the phenomenon of waterborne epidemics in some parts of the globe still persists (Crocker et al., 2017). There is a plethora of knowledge on basic sanitation related to an unhygienic environment; however, there is paucity that links behavioural change among communities to the extent the change influences the effective use of sanitation facilities such as latrines and toilets. Most of the programs offered by health agencies and organisations 
concentrate on funding the construction of sanitation facilities such as latrines and hand washing gadgets, disregarding proper usage. Noteworthy, the provision and construction of sanitation facilities such as latrines and handwashing sites are not enough without proper education on its usage (Ghosh \& Cairncross, 2014; Venkataramanan et al., 2017). Lack of understanding on the use of sanitary facilities stymied effective promotion of basic hygiene at microscale level despite wielded efforts by health promoters.

Therefore, in this study, we debunked the ideas and theoretical concepts based on induced behaviour change from Bandura's socio-cognitive approach. Bandura's social concept postulates that the change of human behaviours is dictated by contact and interaction with environmental components and events (Bandura, 1986). In the context of this study, the information transfer through hearing and observation tends to acts as a major driving force for human behaviour change, particularly in regards to learning processes of individuals and/or community. For instance, the perceptions of resource use, cultures, behaviours and/or community norms (Bandura, 1986). That is, any new knowledge or innovation introduced to any society functions based on the community's own diffusion network for ideas and their interaction and penetration (Tessema, 2017). However, the learned behaviours and/or habits diffused to the population vary depending on the nature and inherent behaviours of the community, which are either extrinsic or intrinsic factors (Izua et al., 2015 \& Tessema, 2017). For instance, community learn or copy common and beneficial practices from their neighbourhood or disseminator.

Intricate behaviour in the context of this study is that the rooted norms among the entire population and/or the neighbourhood in the community are commonly learned over time. This implies that behaviour learned since childhood such as unsafe excreta disposal are transferred to the generation in that community which ultimately become common behaviour among the population (Crocker et al., 2017; Tessema, 2017). Therefore, when new ideas and/or knowledge are learned and transferred or copied through neighbour's practices, when a provided community perceives new idea as common and positive, then it greatly influences the adoption of the new practices with ease by majority members in the community. However, external environment factors influence personal character, which sometimes creates barriers for the adoption of the ideas (Crocker et al., 2017). External barriers that influence knowledge adoption include culture, norms, socio-economics and physical characteristics of the environment (Izua et al., 2015).

Further, an inherent trait in humans portrays the capability of responding to society disorders by reaction, which brings imbalance into normalcy. A society disorder in the context of this study is the compromised unsafe excreta disposal and poor water quality as a result of open defecation practices. In the process of rectifying the imbalance, people tend to transform by passing information, experiences, knowledge and skills through different dissemination pathways (Crocker et al., 2017). As a result, people could expand their knowledge based on strategies made relating to causal effects and externalities. Therefore, conceptualising rational thinking of humans at an individual level and/or collectively in society in response to negative consequences can be expressed in a functional form as $\mathrm{V}(\bullet)$ which represent an indirect environmental utility. Utility in context to this study was the environmental sanitation. This theory holds the assumption that household members follow the axiom of rationality when the right information is transferred to the society.

To achieve effective utilisation of common property by the community, each household ought to invest in health structures and positive practices such as constructing latrines and safe human excreta disposal, respectively. Assuming that households' investment in health facilities to represent environmental utility, then, a direct utility function can be defined over the investment; hence will have the vector $\mathrm{x}$, and $\mathrm{q}$, respectively. The vector relationship of the two variables can be expressed in the utility functional equation as $\mathrm{u}(\mathrm{x}, \mathrm{q})$. The corresponding characteristic from this direct utility in context to consumptive function, it takes a quasiconcave curve in $\mathrm{x}$ and depiction of proportional increases. So that, if the household regards $q$ as a "positive investment," u (x, q) will both be increasing in q; and if the household regards it as a 
"negative investment," $u(x, q)$ will be decreasing in $\mathrm{q}$; while if it is indifferent to $\mathrm{q}, \mathrm{u}(\mathrm{x}, \mathrm{q})$ then it will be independent of q. However, from the aforementioned utility functions, expressed actions to mitigate health vices by individuals and/or society don't always reflect monotonic norms.

Therefore, the axiom of rationality in a community common good remains too abstract (Crocker et al., 2017). That is, at times, people often misread events through cognitive biases in a way that gives rise to faulty beliefs about themselves and the environment around them. When they act on their misconceptions, which appear subjectively rational to them, others will view them as behaving in an unreasonable or foolish manner. Accordingly, supposing society reaction to health investment $\mathrm{q}$ changes from $\mathrm{q}^{0}$ to $\mathrm{q}^{1}$; then, the household's utility will change from $u^{0}=\left(x, q^{0}\right)$ to $u^{1}=\left(x, q^{1}\right)$. If the households regard health investments as an improvement, then $\mathrm{u}^{1}>\mathrm{u}^{0}$ will express positive while the reverse will be expressed as $u^{1}<u^{0}$. When there is indifference from health investment, then the utility will be expressed as $u^{1}=u^{0}$. A change from a situation of high prevalence to a dramatic decrease of waterborne diseases when society reacts through behavioural change shows a paradigm shift of health improvement or willingness to pay attribute.

Incidences of waterborne diseases in the study area still occur at the onset of every rainy season. In 2009, a serious cholera outbreak occurred in the month of April to June as a result of heavy rains which caused many deaths in the study area. The identified cause was established to be nonutilisation of already constructed sanitary facilities such as pit latrines which were funded by health promoters in the region. Despite marshalled efforts by several health stakeholders in funding water and sanitation program (WASH), open defecation still remain the most used excreta disposal means by the communities. As a result, water-related diseases remain rampant in the study area. After the reoccurrence of these environmental episodes which was found to be abetted by unhygienic practices by communities, a program was established by the government jointly with interested organisations in the county in the subsequent year to impart health information. The aim was to induce change behaviour. The use of crude language to the population referred to as "trigger" was an innovative way of bringing awareness aimed to change monotonic habit among communities in the study area to stop defecating in the open. The cognitive approach used was distasteful language aimed at changing the community mindset positively to adopt safe excreta disposal as a mechanism strived to achieve environmental hygiene measures. That is the assumption was that induced behaviour influences society to think over the "bad" happenings in the society, such as the consequences of the environmental problem of unsafe excreta disposals. Several studies have used a similar approach coined as total led sanitation (Izua et al., 2015; Crocker et al., 2017; Venkataramanan et al., 2017).

The triggered information used by the community to break the silence and adopt hygiene practices of latrine construction and use to curtail the environmental disorder was the use of crude, distractible and explicit language that exposes unhygienic community norms and taboos, awful, shame and humiliation around the human waste. As explained by Tessema (2017), the use of the cognitive approach emphasises that, if the minority in the community still defecate in the open, instead of using a latrine, then all members of the community are 'eating and drinking their shit' when flash flood from open defecated areas washes human excreta during the rainy season to the water drawing point. Therefore, this induced unpleasant scenario to members of the community could break the silence by changing the behaviour of the community to observe safe excreta disposal. Based on that, this study sought to compare the effectiveness of induced behavioural change in the "triggered" and "non-triggered" villages.

\section{MATERIALS AND METHODS}

\section{Description of the Study Area}

This survey was carried out in the Central district of Turkana County in Kenya in the month of January to April in the year 2019. The geographical land of the study area is $68,680.3 \mathrm{~km}^{2}$, while latitudes and longitudes lie between $1^{0} 30^{\prime}$ and $5^{\circ} 30^{\prime}$ North, and $34^{\circ} 30^{\prime}$ and $36^{\circ} 40^{\prime}$ East, respectively. The altitude ranges between 360 to 914 metres above sea level where Lake Turkana is at a low level while the 
western part has high elevation with mountain ranges. The county receives between $150 \mathrm{~mm}$ and $400 \mathrm{~mm}$ of rain annually, with average rainfall being $250 \mathrm{~mm}$. The rainfall pattern in the study area is unpredictable and scarce. However, rainfall is more enhanced in high elevated peripherals areas of the study area, especially the landscape of western parts which often cause flash floods at lower plains.

Again, the study area depicts extremely high temperatures throughout the year that range between $21^{\circ} \mathrm{C}$ and $41^{\circ} \mathrm{C}$ with a mean of $30.5^{\circ} \mathrm{C}$. Due to the low rainfall and high temperature, there is a lot of surface water evaporation which makes water to be a scarce commodity. Population projection was estimated to be about 24,973 based on Turkana County principle population and growth rate (Kenya Meteorological Department, 2013). The population distributions are sparse, while settlements are in clustered villages near and along the watercourse. The target population is composed of the native community of the Turkana tribe who are pastoralists and they practice mainly the nomadic mode of life. Apart from practising pastoralist, the Turkana community do supplement their incomes with weaving and charcoal burning.

\section{Research Design and Sampling Procedure}

Purposive sampling was used to identify villages that were exposed to triggering which was guided by County health inventory, while systematic simple random sampling was used in administering a structured questionnaire at the household level (Table 1).

Table 1: Selected villages and the number of households

\begin{tabular}{ll|ll}
\hline Triggered Village & & Non-Triggered Village \\
\hline Sampled Village & No of Respondents & Sample Village & No of Respondents \\
\hline Lolupe & 22 & Ng'irokipi & 19 \\
Nayuu & 18 & Nadipoe & 16 \\
Nakuluja & 21 & Narewa & 20 \\
Kakenyuk & 20 & Power & 21 \\
Kadunyangole & 17 & Napuu & 17 \\
Loturerei & 16 & Napucho & 17 \\
Natoot & 18 & Nabulon & 18 \\
\hline Total & $\mathbf{1 3 2}$ & & $\mathbf{1 2 8}$ \\
\hline
\end{tabular}

To achieve randomness in non-triggered villages, sample units were selected using raffling. After identifying sample units from raffles, a reference point was established of which the immediate households from the reference point were chosen as first respondents, while the selection of subsequent respondents followed the right-hand rule method. Sample sizes from each identified sample unit in Table 1 were obtained using the proportionate ratio of the total population. The sampling size required in this study was determined using the sampling frame of 3,163 households (Kenya Meteorological Department, 2013). The achieved sample size for this study of 260 respondents, it was determined using the following formula (Kothari, 2004).

$$
n=\frac{N C^{2}}{C^{2}+(N-1) e^{2}}
$$

Where; $n$-Size of the sample; $N$-Size of population, $C$ - Coefficient of variation at $(95 \%)$ confidence limit, while $e$ is the standard error at $5 \%$ level.

To achieve systematic randomness, subsequent selection of households for an interview after first respondents were picked by following systematic intervals of every third household on the right-hand side. In a situation where the household head was not present or did not qualify the order, then the next household was selected. However, caution was taken to minimise biases by omitting villages that were closer to the triggered villages selected from raffling.

Information sought from respondents includes; excreta disposal practice, construction year of 
toilets if available, socio-economic characteristics of the household such as gender, age, occupation, level of education, excreta disposal method, distance from homestead to water drawing points (shallow wells) and hygiene practices such as handwashing with soap or ash. A pre-test of the questionnaire was done as a validity test. While to achieve ethical consideration in this study, approval from established protocol was sought because humans as subjects were involved before administering the questionnaire. Again, special efforts were made by observing respondents consent in order to minimise social desirability bias, and other forms of bias; an approach that improved the validity and reliability of data collected. Additionally, in order to get reliable information and data, assurance of not using respondent's information for malice but purely for research purpose was clearly spelt. Furthermore, the confidence spelt to respondents to sanctify expressed information, buttressed the reliability of data sought in this study.

\section{RESULTS AND DISCUSSION}

\section{Excreta Disposal Methods and Personal Hygiene}

Responses from a designed question in a questionnaire that asked the household head to identify excreta disposal methods often used by household members revealed various disposal methods (Table 2).

Table 2: Personal hygiene and proportion of household using various excreta disposal method

\begin{tabular}{lll|ll}
\hline & Triggered & & Non-Triggered \\
\cline { 2 - 5 } Sanitation Practices & Frequency & Use & Frequency & Use \\
\hline Excreta Disposal & 42 & $32.10 \%$ & 74 & $57.7 \%$ \\
Use of Bush & 64 & $48.50 \%$ & 16 & $12.6 \%$ \\
Use of latrine & 14 & $10.50 \%$ & 55 & $43.2 \%$ \\
Open defecation & 28 & $21.42 \%$ & 24 & $18.9 \%$ \\
Cat method & & & & \\
Hygiene and access to a latrine & 34 & $26.56 \%$ & 11 & $8.59 \%$ \\
Shared Latrine & 29 & $22.66 \%$ & 5 & $3.91 \%$ \\
Private Latrine & 33 & $24.81 \%$ & 21 & $9.81 \%$ \\
Hand washing after defecating & & & & \\
\hline
\end{tabular}

The popular excreta disposal methods identified from this study include; open defecation in the bush and open land, the Cat method and latrine. Study results in Table 2 further showed high use of latrine $(48.5 \%)$ in the triggered village compared with the non-triggered village $(12.6 \%)$; implying that triggering exercises influenced household's behaviour to adopt safe excreta disposal in latrines. The low use of unsafe excreta disposal methods in the triggered village (use of bush $32.1 \%$, open defecation $10.5 \%$ and cat method $21.42 \%$ ) compared with the non-triggered village (use of bush $57.7 \%$, open defecation $43.2 \%$ and cat method $18.9 \%$ ) from study findings buttresses the merit of triggering as a measure of correcting the environmental disorder.
Also, triggering reveals to have influenced the marginal proportion of households at nearly $25 \%$ to adopt personal hygiene by washing hands after defecation. The numbers of households that use their own latrine or sharing among the community were more in triggered villages than in nontriggered. However, majority of the respondents in focus group discussion contend that scarcity of water, especially in a nomadic lifestyle, makes them disregard personal hygiene. Notwithstanding, the nomadic lifestyle could contribute to unsafe excreta disposal and rare hand washing practices. Exposure to the dangers of unhygienic practices, especially children is supported by past studies (Venkataramanan et al., 2017). The variance from study findings which showed a significant shift in the adoption of safe excreta disposal use in triggered 
and non-triggered villages could be attributed to the induced behaviour that was done.

\section{Social-Economic Characteristic}

To determine the effectiveness of the cognitive approach in mitigating unhygienic behaviour of open defecation, and inferential test for the means of socio-economic variables in the triggered and non-triggered villages was done. The average household population in the triggered and nontriggered had about 7 persons per household depicts the existence of family size in the study area tend to demand sanitation facilities substantially. Again, the average ages in years of respondents interviewed were about 49 and 48 in triggered and non-triggered villages respectively, suggesting existences of rooted culture that could possibly be transferred to community generation. Other studies of Mukherjee (2011) suggested that the elderly population represent society memory. Additionally, the results of a high number of respondents with no formal education infer the existence of a high illiteracy level among the population in the study area.

Table 3: Comparative statistical test of socio-economic characteristics on the influence of induced behavioural change on open defecation

\begin{tabular}{llll|llc}
\hline & \multicolumn{3}{l|}{ Trigged Village } & \multicolumn{3}{|c}{ Non-Triggered Villages } \\
\cline { 2 - 7 } Household Characteristic & Mean & SD & $\boldsymbol{t}$-test & Mean & SD & $\boldsymbol{t}$-test \\
\hline Age & 49.12 & 13.82 & 2.75 & 47.92 & 12.94 & 2.33 \\
Gender & & & & & & \\
Male & 41.52 & 1.98 & 2.09 & 46.08 & 2.17 & 2.63 \\
Female & 89.48 & 2.97 & 1.93 & 79.92 & 3.08 & 2.02 \\
Level of Formal Education & & & & & & \\
No education & 92.01 & 3.02 & $3.51^{*}$ & 97.87 & 3.89 & 2.98 \\
Basic education & 21.34 & 1.95 & $1.64^{*}$ & 17.78 & 2.05 & 1.83 \\
Post-secondary education & 12.01 & 2.41 & $0.98^{*}$ & 8.01 & 1.84 & $0.71^{*}$ \\
Household Population & 6.70 & 2.49 & 3.02 & 7.09 & 2.12 & 2.48 \\
Distance from homes to water points (m) & 27.34 & 2.67 & $1.94^{*}$ & 31.21 & 3.01 & 1.59 \\
Random factor & - & - & $2.56^{*}$ & - & - & $1.46^{*}$ \\
\hline
\end{tabular}

Note: $*=$ Significant at $p<0.1 ; S D=$ Standard Deviation.

Moreover, results from a statistical test (Table 3) showed levels of education variables to have statistical significance to influence households to use latrines in triggered villages. This critical level of education includes; No education at $(t=3.51, p<$ $0.1)$, Basic education at $(t=1.64, p<0.1)$, and Postsecondary education level at $(t=0.98, p<0.1)$ respectively. Again, the statistical test was done to find the relationship between latrine use and the distance to the water points. The results revealed significantly different in the triggered village at $(t=$ $1.94, p<0.1$ ), while in the non-triggered village was indifferent, respectively. This means that triggering merits the community to act decisively by correcting the environmental imbalance. This finding was concomitant with a past study which points out the significance of innovation diffused to the community tends to changes unsustainable or inherent habit in the society (Tessema, 2017).
Strikingly, household heads with post-secondary education level in the non-triggered village were seen to significantly influence the use of latrine at $(t$ $=0.71, p<0.1$ ). A similar study reinforces the fact that formal education is essential for any progressive society; that's exposure to a high degree of literacy brings developmental progress to society (Nilanjana, 2011). The positive relationship between a household's education level and sanitation status from the results depicts access to formal education tends to uplift individual or household members into a higher position of the sanitation ladder. Therefore, formal education in any society acts as a catalyst to development.

Again, based on the findings, it can be postulated that induced knowledge to the population could reduce water pollution caused by open defecation. When open defecation is reduced, it will enhance 
water security, human dignity and related environmental costs such as waterborne diseases. Notably, the significance of stochastic variable at $(t$ $=2.56, p<0.1)$ and $(t=1.46, p<0.1)$ for triggered and non-triggered villages respectively, explains the existences of underlying variables that remained omitted and/or unobserved in the OLS regression model and were critical in influencing the desire of the community to practice safe excreta.

\section{Disseminating Pathways that Promotes Basic Sanitation}

Information to the community can be disseminated through various dissemination models which include simple and sophisticated ones; however, some of these approaches are more effective than others. In this study, several knowledge dissemination pathways were identified. The findings showed that Public health meetings with the community $(t=3.11, p<0.1)$ and neighbourhood and/or friends $(t=2.73, p<0.1)$ (see Table 4) to significantly influence the population to upscale basic sanitation in triggered villages. It could be seen from the results that expert knowledge remains important in information dissemination platform, while the relevance of neighbourhood and friends comes from the frequency of meeting; that's closer information sources tend to influence knowledge adoption (Crocker et al., 2017). That is

when an idea is introduced from neighbourhood or friends from constant interaction; they replicate action by the majority at their homes, which subsequently have a multiplier effect that will eventually change the behaviour of the entire community (Tessema, 2017; Crocker et al., 2017). However, concerns were raised by respondents on other disseminating means (written materials and visual) by citing inability by the majority of the respondents to read. Although the majority claimed to have a portable radio, they cited that other programs of sanitation were less pronounced, especially in their native language for them to comprehend well.

Surprisingly, the majority cited the inability of an opinion leader's to explicitly explain health issues which complicated the understandings of health issues, hence, a conflicting view. Hence, reliance on expert's knowledge in disseminating health information remains critical. However, the significance of random factor at $(t=2.56, p<0.1)$ and $(t=1.46, p<0.1)$ (see Table 4$)$ for triggered and non-triggered villages, respectively, which explains omitted and/or unobserved variables in the model, informs the existences of underlying critical disseminating approaches. Hence, such unexplained variables in the models form part of the limitation of this study.

Table 4: Categories of Information Dissemination Pathways used in Promoting Basic Sanitation

\begin{tabular}{llll|lll}
\hline Information Dissemination & \multicolumn{3}{l}{ Triggered Village } & & \multicolumn{2}{l}{ Non-Triggered Villages } \\
\cline { 2 - 7 } Pathways & Use & f & $\boldsymbol{t}$-test & Use & $\boldsymbol{f}$ & $\boldsymbol{t}$-test \\
\hline Public Health Meetings & Moderately & 97 & $3.11^{*}$ & Moderately & 86 & 2.72 \\
Leaders Forums (Chiefs and elders) & Mostly & 75 & 2.08 & Mostly & 81 & 1.99 \\
Written pamphlets and newspapers & Rarely & 103 & 1.97 & Rarely & 99 & 2.18 \\
Audio means (Radio) & Rarely & 112 & 2.70 & Rarely & 107 & 2.31 \\
Visual Means (Television) & Not at all & 117 & 0.85 & Not at all & 121 & 1.02 \\
Neighbourhood and/or Friends & Moderately & 125 & $2.73^{*}$ & Moderately & 119 & 2.24 \\
Random factor & - & - & $3.26^{*}$ & - & - & $1.98^{*}$ \\
\hline
\end{tabular}

Note: $*$ Significant at $p<0.1 ; H H=$ Household; SD = Standard Deviation; Popularity of

dissemination pathways was tested using 4 scale scoring (most, moderate, rarely and Not at all).

\section{Latrine Construction as Adoption Response Measure}

Figure 2 shows comparative cumulative numbers of latrines between triggered and non-triggered villages constructed between the year of 2010 to 2019 for either individual household and/or community in the study area. 
Figure 1: Graph showing cumulative numbers of constructed latrines from 2010 to 2019 at

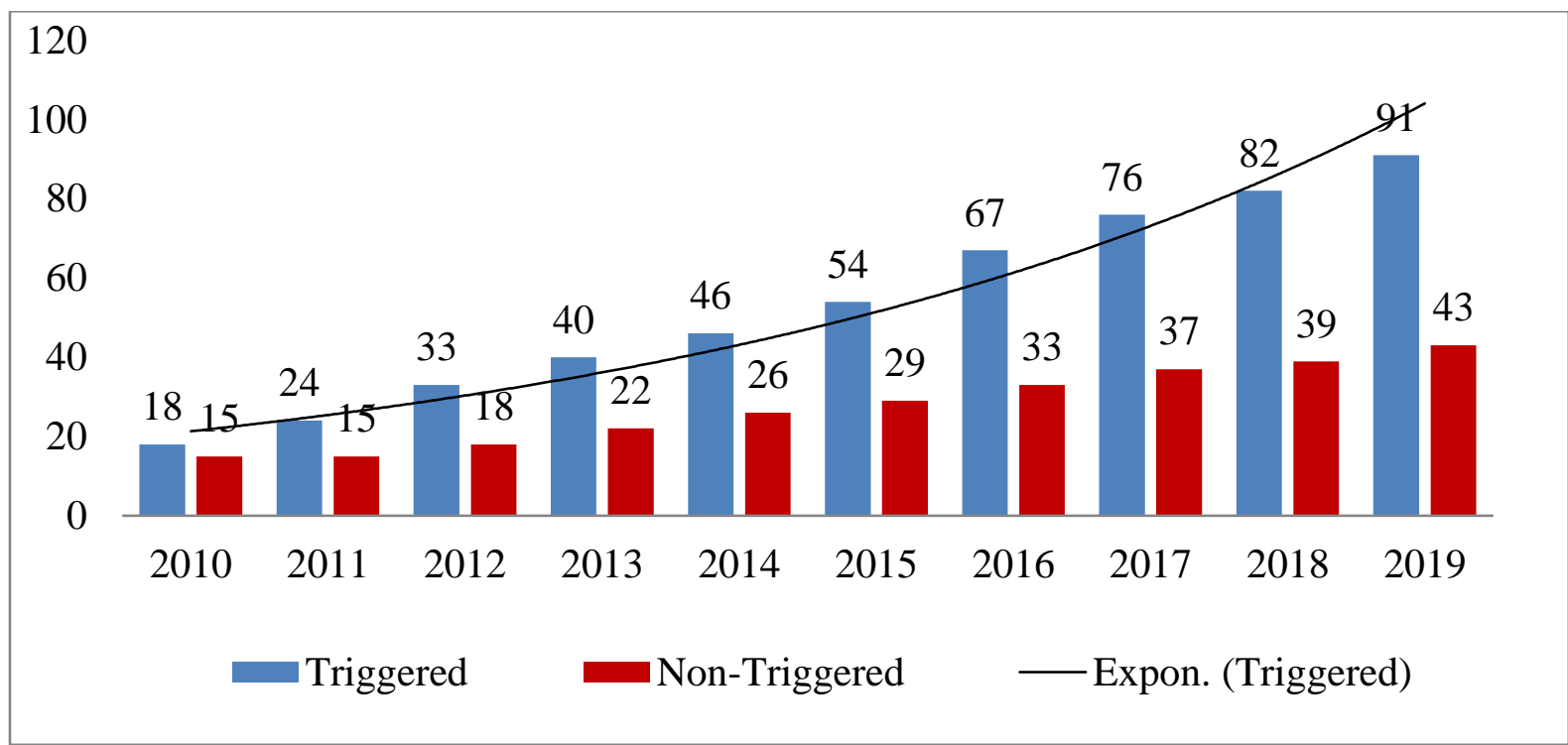

Existences of variance in latrine use and ownership in both triggered and non-triggered villages as depicted by study results reveal the significance of induced sanitation knowledge in reducing unsafe excreta disposal to the community. That's a high exponential rate of increase in the construction of new latrines at triggered villages than in nontriggered villages depicts the effectiveness of triggering. Of interest was the constructed latrines in triggered villages were being owned by individual households, while in non-triggered villages majority of latrines were government or Nongovernmental funded. This suggests households' expressed willingness to upscale sanitation in terms of costs incurred in constructing private latrine to avoid shame, environmental cost and/or improve water quality.

Despite the exponential increase of latrine construction in triggered villages, open defecation as pointed out by focus group discussion still stubbornly persist, which makes triggering practice an inconclusive measure. This could inform the need to mix various approaches as a strategy that reduces unsafe excreta disposal. A review study (O'Connell, 2014) on open defecation in rural areas showed that household ownership of latrine, or access to the shared latrine in community, does not guarantee the utilisation or consistently use by household members which make ownership of sanitary facility as a non-reflective factor. Although other barriers were not investigated in this study, such omitted obstacles form part of the limitation in this study; a platform that informs future investigation. Other studies (O'Connell, 2014; Crocker et al., 2017) identified several barriers that bulk the proper use of latrines in rural areas. These barriers include comfortability, beliefs related to latrines, distance to the facility, safety and misuse, especially on the shared latrine.

\section{CONCLUSION AND RECOMMENDATIONS}

Provision of public health concerns through crude form showed to effectively raise sanitation awareness among rural communities. The findings of this study showed that induced behaviour to the community changed human mindset at large scale perception in excreta management. The induced behaviour showed to have a high propensity to transform the significantly retrogressive attitude of unused latrine by the community by reverting their crude way of managing human waste. Further, study findings revealed an exponential increase in the construction of latrines in places where triggering took place, suggesting induced behaviour to have raised awareness levels among the community to build their latrines. Hence, triggering is a reflection of the willingness to pay attributes.

Despite the significance of triggering through behavioural inducement, which influenced 
community attitude to adopt construction latrines from study result, there exist compromises from the approach that bulk its full-scale achievements in environmental sanitation. Probable factors that were beyond the scope of this study include; nomadic lifestyle, high illiteracy level, generational culture and norms among the community. With that notwithstanding, perhaps, formal education among the community revealed to be a likely long-term solution in reducing the prevalence of unsafe excreta disposal in the study area.

Again, knowledge diffusion and training to the community points out the need to engage health experts in disseminating health information in order to make the community understand the basics. Therefore, training of more public health extension practitioners to impart knowledge using triggering techniques is recommended. This could motivate the community to intercepts the right health information, which gets diffused evenly from interceptors to the community through dissemination means of neighbours and friends. Information diffusion networks could probably have multipliers effects in influencing most households in the community to adopt safe excreta disposal. The existence of the study limitation informs the need for further studies. Further investigation needs to be done to uncover positive externalities that are associated with latrine construction, use and impact on disease prevalence in the study area.

\section{ACKNOWLEDGMENTS}

We thank respondents and Turkana County Public Health Department for their valuable information they contributed, which made this survey successful.

\section{REFERENCES}

Bandura, A. (1986). Social Foundation of Thought and Action - A Social Cognitive Theory. New Jersey: Premise-Hall.

Crocker et al., J., Saywell, D., \& Bartram, J. (2017). Sustainability of community-led total sanitation outcomes: Evidence from Ethiopia and Ghana. International Journal of Hygiene and Environmental Health, 220(3), 551-557.
Ghosh, A., \& Cairncross, S. (2014). The uneven progress of sanitation in India. Journal of Water, Sanitation and Hygiene for Development, 4(1), $15-22$.

Izua, M.L., Djebbri, H., Lopez, C., Cardenas, J.C., Lopera, M. A., Osbert, N., \& Coulibaly, M. (2015). Final report: Impact evaluation of Community-Led Total Sanitation in rural Mali. Geneva: UNICEF.

Kenya Meteorological Department. (2013). The outlook for the March-April-May (MAM) 2013 "long-Rains" season in Kenya and review of the performance of the October-December 2012 "Short Rains" Seasons as well as the weather during January-February 2013. Report from Government of Kenya [online]. Kenya Meteorological Department

Kothari, C. R. (2004). Research Methodology, methods and techniques ( $2^{\text {nd }}$ Ed.). New Age International (p) Ltd. Publishers, New Delhi.

Mukherjee, N. (2011). Factors associated with achieving and sustaining open defecation free communities: Learning from east java. Water and sanitation program, 2011, 1-8. Pearce D.W. and Turner R.K. (1990). Economics of Natural Resources and the Environment. Harvester Wheatsheaf. New York.

O'Connell, K. A. (2014). What influences open defecation and latrine ownership in rural households? Findings from a global review (No. 90044, pp. 1-38). The World Bank.

Tessema, R. A. (2017). Assessment of the implementation of community-led total sanitation, hygiene, and associated factors in Diretiyara district, Eastern Ethiopia. PloS one, 12(4), e 0175233.

Venkataramanan, V. (2017). Review of Rural Sanitation Approaches. Final Report. Mulugeta Balecha. The Water Institute.

WHO and UNICEF. (2014). UNICEF Joint Monitoring Programme Database. Retrieved from http://www.wssinfo.org/

WSP, IFC \& MoHK. (2013). Water and Sanitation Program. End of Year Report, Fiscal Year 
East African Journal of Health and Science, Volume 3, Issue 1, 2021

Article DOI: https://doi.org/10.37284/eajhs.3.1.294

2013. Water and Sanitation Program (WSP), International Finance Corporation (IFC) \& Ministry of Health, Kenya (MoHK). Retrieved from https://www.wsp.org/sites/wsp/files/public ations/WSP-End-Year-Report-FY13.pdf. 